\title{
PLANTA DIDÁTICA SMAR PD3: MODELAGEM E SIMULAÇÃO DO TANQUE DE AQUECIMENTO - Parte A
}

\author{
C. R. R. de MORAIS ${ }^{1}$, N. B. DOMINGOS ${ }^{2}$, J. V. NICACIO ${ }^{3}$ e A. G. TÔRRES ${ }^{4}$ \\ ${ }^{1}$ Universidade Federal de Viçosa, Departamento de Química \\ ${ }^{2}$ Universidade Federal de Viçosa, Departamento de Química \\ ${ }^{3}$ Universidade Federal de Viçosa, Departamento de Engenharia Agrícola e Ambiental \\ ${ }^{4}$ Universidade Federal de Viçosa, Departamento de Engenharia Elétrica \\ E-mail: camilamorais.cr@gmail.com
}

\begin{abstract}
RESUMO: $O$ controle PID (proporcional, integral e derivativo) é importante para assegurar a padronização e segurança de diversos processos químicos de uma indústria, assim como a qualidade de seus produtos. Neste trabalho foram realizadas as modelagens matemáticas teórica e experimental do tanque de aquecimento da planta didática III (PD3) da Smar para posterior sintonia do controlador a ser realizada na parte B deste estudo. A Planta didática está localizada no Laboratório de Pesquisa de Processos Industriais e Padrões de Movimentos e Acústica do Departamento de Engenharia Elétrica da Universidade Federal de Viçosa. Para a modelagem foi necessário obter os parâmetros da função de transferência do processo de aquecimento do tanque em questão, utilizando degraus de potência para vazões constantes de 200, 400, 600 e 1000 L.h. ${ }^{-1}$ e análise dos dados fornecidos pelo programa Process View. Os resultados experimentais foram consistentes com os teóricos, a diferença dos ganhos foi de no máximo 26,3\% e das constantes do tempo foi de 10 a 15\%, corroborando a eficiência da metodologia aplicada.
\end{abstract}

PALAVRAS-CHAVE: Modelagem; Processos Químicos; Função de Transferência; Controlador PID.

\section{INTRODUÇÃO}

A performance requerida nas plantas de processos tem se tornado cada vez mais difícil de satisfazer. A alta competitividade do mercado, regulamentações ambientais e de segurança e as rápidas mudanças nas condições econômicas tem sido fatores determinantes no afunilamento das especificações de qualidade. Uma posterior complicação é que plantas modernas tem se tornado mais difíceis de operar devido à tendência de processos cada vez mais complexos e altamente integrados. Para tais plantas, é difícil prevenir que distúrbios sejam propagados de uma unidade para as outras interconectadas.

Tendo em vista o aumento da ênfase colocada em operações de plantas de forma segura e eficiente, o interesse em controle de processos se tornou mais importante há alguns anos. Sem sistemas de controle de processos automatizados seria impossível operar plantas 
modernas de forma segura e rentável, ainda satisfazendo a qualidade do produto e requisitos ambientais.

Para se obter sucesso no controle de um sistema é necessário o estudo da dinâmica do processo, sendo que em primeiro lugar deve-se conhecer o comportamento dinâmico do sistema sem auxílio ou interferência do controlador (KWONG, 2012). Além disso, de acordo com Ahmed, Huang e Shah (2008), para entender a resposta transiente de sistemas de diversas ordens é possível aplicar um distúrbio no processo e analisar a reposta obtida. Dessa forma, é possível fazer a modelagem do sistema e posteriormente definir a melhor estratégia de controle para esse determinado processo.

À vista disso, o objetivo deste trabalho foi realizar a modelagem do tanque de aquecimento da planta Smar PD3 com o estudo da resposta transiente ao degrau de potência para vazões constantes de 200, 400, 600 e 1000 L.h ${ }^{-1}$.

\section{REVISÃO DE LITERATURA}

\subsection{Planta Didática}

A planta didática SMAR tem o objetivo de demonstrar de forma didática a operação das diversas malhas de controle utilizando equipamentos e ferramentas de configuração similares àqueles de aplicação de controle industrial. A planta foi desenvolvida com o objetivo de ser manipulada, sendo que é acessível devido ao seu arranjo compacto. $\mathrm{Na}$ implementação destas malhas estão englobadas as mesmas características e situações observadas em contextos industriais, com recursos de alta tecnologia (SMAR, 2012).

\subsection{Modelagem de Sistemas}

No projeto de controladores para processos químicos, é necessário o conhecimento da representação matemática dos fenômenos físicos e químicos que ocorrem em tais processos. Esta representação constitui o modelo do sistema, e a tarefa de construir o modelo chama-se modelagem (KWONG, 2012).

De acordo com Seborg, Edgar e Mellichamp (2003), os modelos matemáticos podem ser classificados de acordo com sua forma de obtenção, sendo eles os modelos teórico, experimental e híbrido. Os modelos teóricos são desenvolvidos utilizando princípios da química, física e biologia. Os modelos experimentais são obtidos com o ajuste de dados experimentais. Por fim, os modelos híbridos são a combinação dos modelos teóricos e experimentais, sendo que os valores numéricos de um ou mais parâmetros do modelo teórico são calculados a partir de dados experimentais.

Modelos teóricos oferecem duas importantes vantagens: eles fornecem a compreensão do comportamento do processo e eles são aplicáveis em amplas faixas de condições. Porém, existem desvantagens associadas a modelos teóricos. Eles têm a tendência de serem caros e demoram para serem desenvolvidos. Além disso, modelos teóricos de processos complexos normalmente incluem alguns parâmetros do modelo que não estão prontamente disponíveis, 
como coeficientes de taxa de reações, propriedades físicas ou coeficientes de transferência de calor (SEBORG, EDGAR, MELLICHAMP, 2003).

Os modelos experimentais são mais fáceis de obter do que modelos teóricos, porém, eles têm sérias desvantagens: dependem de que já se possua o processo ou equipamento construído e geralmente não podem ser extrapolados com precisão. Mais especificamente, modelos experimentais devem ser usados com cuidado para operações com condições que não foram incluídas nos dados experimentais usados para ajustar o modelo. $\mathrm{O}$ alcance dos dados é geralmente bem pequeno comparado com a amplitude total das condições das operações do processo (SEBORG, EDGAR, MELLICHAMP, 2003).

Os modelos híbridos possuem três vantagens inerentes: eles incorporam o conhecimento teórico, podem ser extrapolados através de uma maior variedade de condições de operação do que o modelo empírico e, por fim, eles requerem menos esforço para desenvolver do que modelos teóricos. Consequentemente, modelos híbridos são amplamente utilizados na indústria (SEBORG, EDGAR, MELLICHAMP, 2003).

Em todos os casos é encontrada uma função de transferência que descreve a dinâmica do processo. Na teoria de controle as funções de transferência são usadas para caracterizar as relações de entrada e saída de componentes ou sistemas descritos por equações diferenciais lineares invariantes no tempo. Ela é definida como a transformada de Laplace da saída, função de resposta, dividida pela transformada de Laplace da entrada, função de excitação (OGATA, 2010). A Equação 1 apresenta a função de transferência de um processo genérico, segundo Skoczowski (1997), com entrada e saída simples:

$$
G(s)=\frac{b}{a_{n} s^{n}+a_{n-1} s^{n-1}+\cdots+a_{1} s+a_{0}}
$$

A dinâmica do sistema será representada por uma equação algébrica em S. Para determinar a ordem do sistema é necessário olhar a maior potência do denominador da função de transferência. Deste modo, se a maior potência do denominador for igual a $\mathrm{n}$, o sistema terá ordem n (OGATA, 2010).

Um sistema de primeira ordem é aquele cuja saída é modelada por uma equação diferencial ordinária de primeira ordem.

Logo, tem-se a forma padrão de um sistema de primeira ordem:

Erro! Fonte de referência não encontrada.

Onde:

Erro! Fonte de referência não encontrada. é a constante de tempo do processo e tem as dimensões de tempo, caracteriza a velocidade com que o sistema responde a uma certa entrada.

Erro! Fonte de referência não encontrada. é o ganho do processo em regime permanente de operação.

Aplicando a transformada de Laplace na Equação 2 e rearranjando, tem-se: 


$$
G(s)=\frac{K_{p}}{\tau_{p} s+1}
$$

Que é a função de transferência de um sistema de primeira ordem.

Um sistema de segunda ordem é aquele cuja saída é modelada por uma equação diferencial de segunda ordem, sendo que sua forma padrão é:

Erro! Fonte de referência não

\section{encontrada.}

Onde:

Erro! Fonte de referência não encontrada. é o tempo característico ou período natural de oscilação do sistema, o qual determina o tempo de resposta do sistema.

$\zeta$ é o fator de amortecimento, adimensional, que dá a medida da quantidade de amortecimento do sistema, ou seja, o grau de oscilação na resposta do processo após uma perturbação.

Erro! Fonte de referência não encontrada. é o ganho do sistema.

Aplicando a transformada de Laplace na Equação 4 e rearranjando, tem-se:

$$
G(s)=\frac{K_{p}}{\tau^{2} s^{2}+2 \zeta \tau s+1}
$$

Onde a Equação 5 é a função de transferência de um sistema de segunda ordem.

Características dos Sistemas de Primeira Ordem: Processos de primeira ordem são caracterizados por sua capacidade de armazenar materiais, energia e momento, além de resistência associada com o fluxo de massa, energia ou momento para atingir a capacidade do sistema (STEPHANOPOULOS, 1984).

Desta maneira, a resposta dinâmica de tanques que possuem a capacidade de armazenar líquidos ou gases pode ser modelada como primeira ordem. A resistência é associada com bombas, válvulas e tubulações que são ligados aos fluxos de entrada e saída de líquidos e gases. De forma similar, a resposta da temperatura de sistemas sólidos, líquidos e gasosos que tem a capacidade de armazenar energia térmica é modelada como de primeira ordem. Para tais sistemas a resistência é associada com a transferência de calor através das paredes, líquidos ou gases (STEPHANOPOULOS, 1984).

Resposta Transiente de Sistemas de Primeira Ordem: Pode-se aplicar diversos sinais de entrada em determinado processo, os quais possuem várias maneiras de ocorrer e de serem classificados. Com relação à forma, os sinais de entrada podem ser classificados como degrau, pulso, impulso, rampa e senoidal (LUYBEN, 1996). 
As perturbações em degrau são funções que modificam instantaneamente de um nível ao outro e depois se torna constante. O degrau unitário é aquele com a amplitude igual a unidade (LUYBEN, 1996).

Sabe-se que a representação matemática de uma variação degrau de amplitude A é:

Erro! Fonte de referência não encontrada.

Onde Erro! Fonte de referência não encontrada. é a função degrau unitário.

Aplicando a transformada de Laplace da Equação 6, tem-se a Equação 7:

$$
f(s)=\frac{A}{s}
$$

Para sistemas de primeira ordem, aplicando a Equação 7 na Equação 3 tem-se que:

$$
Y(s)=\frac{A K_{p}}{s\left(\tau_{p} s+1\right)}
$$

Aplicando a transformada inversa, obtém-se a seguinte resposta y(t):

$$
y(t)=A K_{p}\left(1-e^{-t / \tau_{p}}\right)
$$

Um aspecto importante da resposta de um sistema de primeira ordem ao degrau é que o valor de y(t) alcança 63,2\% de seu valor final após decorrido um intervalo de tempo igual a uma constante de tempo.

Modelagem Teórica: Modelos de processos químicos dinâmicos consistem de equações diferenciais ordinárias (EDO) e/ou equações diferenciais parciais (EDP), mais relações algébricas relacionadas. Para problemas de controle de processos, modelos dinâmicos são derivados usando leis de conservação do estado permanente. As equações algébricas, que geralmente aparecem nos modelos, provêm de considerações de termodinâmica, fenômenos de transporte, propriedades físicas e cinética química. Equilíbrio líquido-vapor, correlações de transferência de calor e expressões de taxa de reação são exemplos típicos de tais equações algébricas (SEBORG, EDGAR, MELLICHAMP, 2003).

Transferência de Calor: Incropera e Dewitt (2008, p. 2) mencionaram que "Transferência de calor é a energia térmica em trânsito devido a uma diferença de temperaturas no espaço". Portanto, a perda de calor irá afetar diretamente no ganho da função de transferência do processo, visto que foram utilizados degraus de potência que aumentam ou diminuem os gradientes de temperatura.

Existem três modos de transferência de calor: condução, convecção e radiação. A condução é caracterizada pela transferência de calor através de meios estacionários que possuem um gradiente de temperatura. A convecção se dá através de uma superfície e fluidos 
em movimento quando há diferença de temperatura. Por fim, a radiação térmica acontece com a emissão de ondas eletromagnéticas por qualquer superfície de temperatura não nula.

\section{METODOLOGIA}

Este trabalho foi realizado no Laboratório de Pesquisa de Processos Industriais e Padrões de Movimentos e Acústica do Departamento de Engenharia Elétrica da Universidade Federal de Viçosa.

Para realização deste experimento foi utilizada a planta didática Smar PD3, representada na Figura 1.

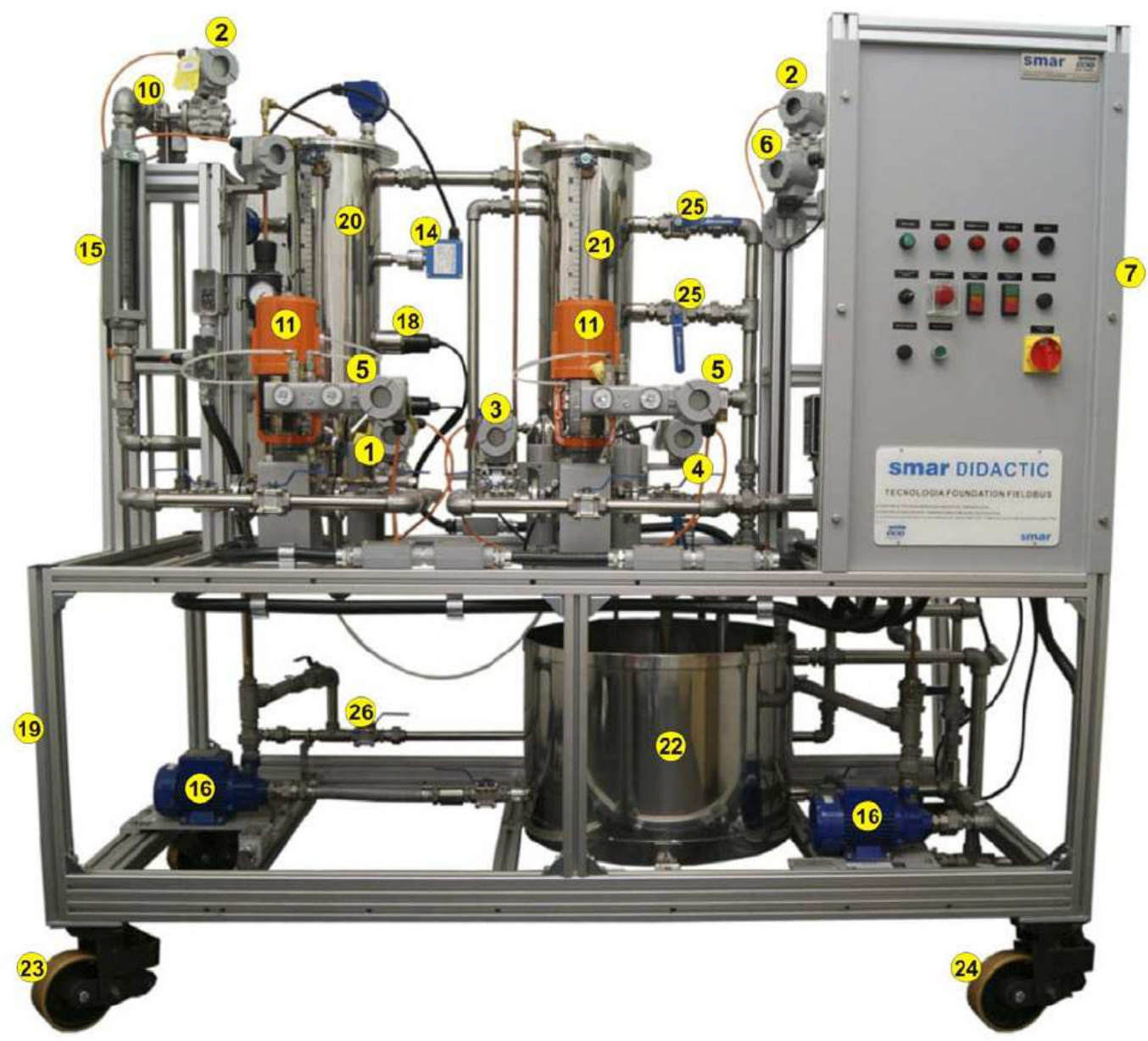

Figura 1 - Vista frontal da planta didática utilizada.

Fonte: Smar, 2012.

Na planta didática a água inicialmente é armazenada no tanque 22 e bombeada pela bomba 16 para o tanque de aquecimento 20. As resistências 18 aquecem a água no tanque 20, onde a vazão de entrada pode ser ajustada pela válvula 1 e observada no rotâmetro 15 . A água quente que deixa o tanque de aquecimento é descartada após passar pelo tanque de mistura 21. 


\subsection{Obtenção da Função de Transferência do Processo}

Os experimentos para modelar o processo de aquecimento foram realizados no tanque 20, que pode ser observado na Figura 1, para quatro vazões distintas, sendo elas de 200, 400, 600 e 1000 L.h ${ }^{-1}$. A escolha dessas vazões se deu devido à necessidade dos testes em uma ampla faixa, sendo que a primeira vazão é próxima da mínima de operação da planta didática, duas seguintes são medianas e a última é próxima da vazão máxima, visto que as indústrias vão operar, na maioria das vezes, em suas capacidades máxima de produção.

Para iniciar os experimentos foi necessário esperar a temperatura inicial entrar no regime permanente. Quando o sistema estabilizou, aplicou-se no conversor de potência o sinal degrau de corrente nos valores de 8, 12 e $20 \mathrm{~mA}$, por um determinado tempo, que correspondem a um sinal degrau de potência de 2,4 e $8 \mathrm{KW}$ respectivamente. Para vazão de 1000 L.h $^{-1}$ aplicou-se somente o degrau de $8 \mathrm{KW}$, pois, devido à alta vazão de água, as potências de 2 e de $4 \mathrm{KW}$ aqueceriam muito pouco a água.

O tempo de aplicação de cada degrau de potência foi aquele suficiente para que o sistema entrasse em regime permanente em cada experimento, ou seja, até que a temperatura dentro do tanque se tornasse constante.

O objetivo dos experimentos foi obter a curva de resposta da temperatura durante o processo de aquecimento no tanque de água quente. Dessa forma, foi possível calcular o ganho do processo e o valor da constante de tempo, obtendo-se a função de transferência de primeira ordem do processo. O programa da planta didática, ProcessView, gera um arquivo em formato csv com todas as temperaturas medidas a cada segundo, sendo que esse arquivo foi tratado e organizado para se obter as temperaturas em ordem crescente. Esses valores de temperatura foram copiados para o Matlab®, onde uma matriz foi criada e em seguida subtraída do valor no regime permanente inicial do experimento, para que todas as variáveis estivessem no formato de variável desvio e por meio da função "Plot(y)" foram obtidas as curvas de medição da temperatura.

\subsection{Modelagem Teórica}

A modelagem teórica deste trabalho foi feita utilizando balanços de massa e energia para um tanque de aquecimento cilíndrico e sem agitação, representado na Figura 2, conforme pode ser vista com mais detalhes em Kwong (2012, v. 1, p. 35). 


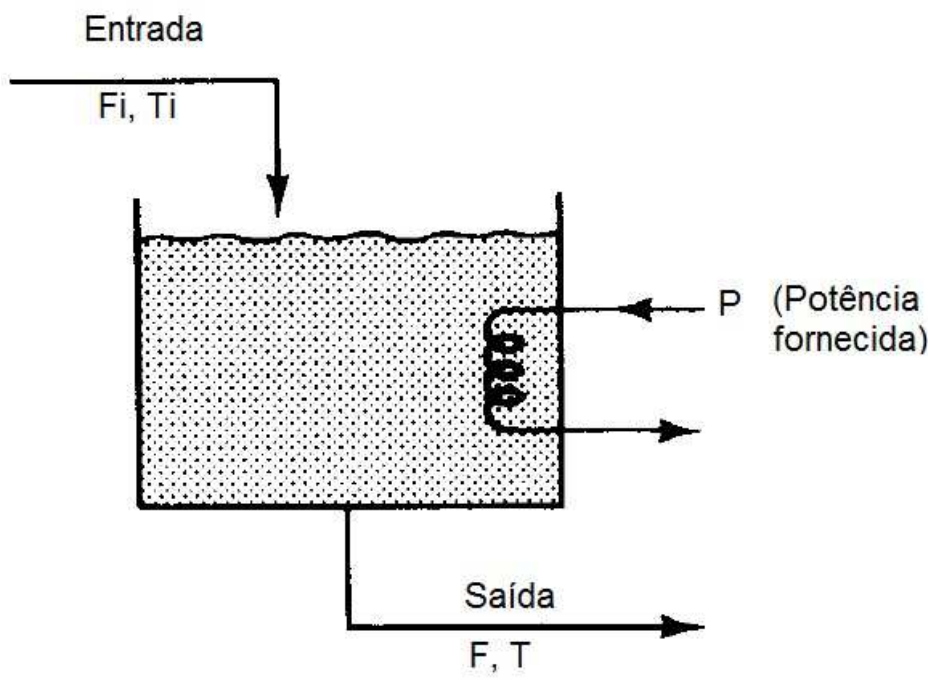

Figura 2 - Representação do tanque de aquecimento sem agitação.

O balanço de massa do processo é descrito abaixo:

$\frac{d(\rho A h)}{d t}=\rho F_{i}-\rho F$

$A \frac{d h}{d t}=F_{i}-F$

O balanço de energia tem a seguinte forma geral:

$\frac{d E}{d t}=\frac{d(U+K+P)}{d t}=\sum_{\text {entrada }} \rho_{i} F_{i} h_{i}-\sum_{\text {saida }} \rho_{j} F_{j} h_{j} \pm q^{n} \pm W_{s}$

Onde:

Erro! Fonte de referência não encontrada. é a densidade;

Erro! Fonte de referência não encontrada. é a área da base do reservatório;

Erro! Fonte de referência não encontrada. é a altura do reservatório;

Erro! Fonte de referência não encontrada. é a energia interna do sistema;Erro! Fonte de referência não encontrada.

Erro! Fonte de referência não encontrada. é a energia potencial do sistema;

Erro! Fonte de referência não encontrada. é a energia cinética do sistema;

Erro! Fonte de referência não encontrada. é a vazão volumétrica do fluido; 
Erro! Fonte de referência não encontrada. é a entalpia específica do material de entrada;

Erro! Fonte de referência não encontrada. é a entalpia específica do material de saída;

Erro! Fonte de referência não encontrada. é a quantidade de calor trocado entre o sistema e as vizinhanças por unidade de tempo;

Erro! Fonte de referência não encontrada. é o trabalho mecânico trocado entre o sistema e as vizinhanças por unidade de tempo.

Não existe energia potencial e cinética, logo:

$\frac{d k}{d t}=\frac{d P}{d t}=0$

E a energia interna pode ser aproximada com o valor da entalpia, assim:

$\frac{d U}{d t} \sim \frac{d H}{d t}$

$H=\rho V C_{p}\left(T-T_{r e f}\right)=\rho A h C_{p}\left(T-T_{\text {ref }}\right)$

Onde:

Erro! Fonte de referência não encontrada. é o volume do reservatório;

Erro! Fonte de referência não encontrada. é a capacidade calorífica da substância;

Erro! Fonte de referência não encontrada. é a temperatura do meio;

Erro! Fonte de referência não encontrada. é a temperatura de referência, que será $0^{\circ} \mathrm{C}$;

Substituindo a Equação 15 na Equação 12:

$\frac{d(\rho A h C p T)}{d t}=\rho_{i} F_{i} C p_{i}\left(T_{i}-T_{r e f}\right)-\rho_{j} F_{j} C p_{j}\left(T_{j}-T_{r e f}\right)+q^{\prime \prime}$

E simplificando dividindo tudo por Erro! Fonte de referência não encontrada.:

$A \frac{d(h T)}{d t}=F_{i} T_{i}-F_{j} T_{j}+\frac{q^{\mathrm{n}}}{\rho C p}$

Fazendo a regra da cadeia e utilizando a Equação 11 para substituir:

$A \frac{d(h T)}{d t}=A h \frac{d T}{d t}+A T \frac{d h}{d t}=F_{i} T_{i}-F_{j} T_{j}+\frac{q^{\prime \prime}}{\rho C p}$

No regime permanente: 


$$
F_{i} T i_{s}-F_{i} T_{s}+\frac{q^{\prime \prime}}{\rho C p}=0
$$

Subtraindo a Equação 19 da Equação 18 para colocar em varáveis desvio, tem-se:

$$
F_{i}\left(T_{i}-T i_{s}\right)-F_{i}\left(T-T_{s}\right)+\frac{\left(q^{\mathrm{H}}-q_{s}^{\mathrm{n}}\right)}{\rho C p}=A h \frac{d\left(T-T_{s}\right)}{d t}
$$

As variáveis desvio são:

$$
\begin{aligned}
& T_{i}-T i_{s}=T_{i}^{\prime} \\
& T-T_{s}=T^{\prime} \\
& q^{\mathrm{n}}-q_{z}^{\mathrm{I}}=P^{\prime}
\end{aligned}
$$

Onde Erro! Fonte de referência não encontrada. e Erro! Fonte de referência não encontrada. representam a potência fornecida que é dada em Watt.

Dessa forma temos:

$$
F_{i} T_{i}^{\prime}-F_{i} T^{\prime}+\frac{P^{\prime}}{\rho C p}=A h \frac{d T^{\prime}}{d t}
$$

Aplicando a Transformada de Laplace na Equação 24:

$$
\begin{aligned}
& F_{i} T_{i}(S)-F_{i} T(S)+\frac{p(S)}{\rho C p}=A h S T(S) \\
& T(S)(A h S+F i)=\frac{P(S)}{\rho C p}+F_{i} T_{i}(S)
\end{aligned}
$$

Como a função de transferência obtida nos experimentos relaciona a temperatura de saída com a potência de entrada, desconsideramos o termo da temperatura de entrada e assim a função de transferência $G_{P}(S)$ é:

$$
G_{p}(S)=\frac{T(S)}{P(S)}=\frac{1}{A h \rho C p S+\rho C p F i}
$$

Colocando ela na forma geral de primeira ordem como mostrado na Equação 3:

$$
G_{p}(S)=\frac{\frac{1}{\rho C p F i}}{\frac{A h \rho C p S}{\rho C p F i}+\frac{\rho C p F t}{\rho C p F i}}=\frac{\frac{1}{\rho C p F i}}{\frac{A h}{F i} S+1}
$$


Portanto:

$$
\begin{aligned}
& K_{p}=\frac{1}{\rho C p F t} \\
& \tau_{p}=\frac{A h}{F i}
\end{aligned}
$$

Onde, a vazão deve ser colocada $\mathrm{em} \mathrm{m}^{3} \cdot \mathrm{s}^{-1}$ e seguindo as características físicas do tanque:

$A=0,034241349 \mathrm{~m}^{2}$

Erro! Fonte de referência não encontrada.

$\rho=1000^{\mathrm{kg}} / \mathrm{m}^{3}$

Erro! Fonte de referência não encontrada.

\subsection{Validação das Funções de Transferência do Processo}

Para validar o modelo foram feitos testes de validação nas vazões testadas, porém com um degrau diferente do que havia sido aplicado nos testes anteriores. Para obter as funções de transferências foram usados testes com degrau de 2, 4 e $8 \mathrm{~kW}$, sendo assim, para validar, foi feito um teste com degrau de $6 \mathrm{~kW}$. Dessa maneira, de posse das funções de transferência média dos testes anteriores, mostradas na Tabela 6 , tidas agora como verdadeiras, comparouse com o novo teste com degrau diferente e com a função de transferência obtida através da Equação 28, substituindo pelos valores mencionados das características físicas do tanque na modelagem teórica.

\section{RESULTADOS E DISCUSSÃO}

\subsection{Obtenção da Função de Transferência do Processo}

A função de transferência descreve completamente o comportamento dinâmico da saída de um sistema quando é dada a mudança correspondente da entrada. Como ela é uma característica do processo, independente da perturbação aplicada, esperou-se que obtivesse funções de transferência iguais ou próximas para o mesmo valor de vazão.

As funções de transferência obtidas do processo são de primeira ordem, ou seja, apresentam o formato mostrado na Equação 3. Estas foram calculadas a partir dos gráficos obtidos da resposta do sistema aos degraus de potência aplicados.

O ganho do processo $(\mathrm{Kp})$ é o valor no regime permanente que a temperatura alcançou depois de dado o degrau, dividido pela amplitude do degrau (quando o mesmo não é 
unitário). A constante de tempo (Erro! Fonte de referência não encontrada.) é o tempo que o sistema leva para atingir $63,2 \%$ do valor da temperatura no regime permanente.

É necessário, também, trabalhar com variáveis desvio, que é o valor da variável subtraído de seu valor no regime permanente. No caso da modelagem do processo, subtrai-se o valor da temperatura da água no tanque em cada instante de tempo pela temperatura inicial da água, pois, dessa forma, todos os resultados encontrados estarão livres da influência dessa temperatura inicial que pode variar para cada teste realizado.

Para uma vazão mínima de 200 L.h ${ }^{-1}$ obteve-se a curva mostrada na Figura 3.

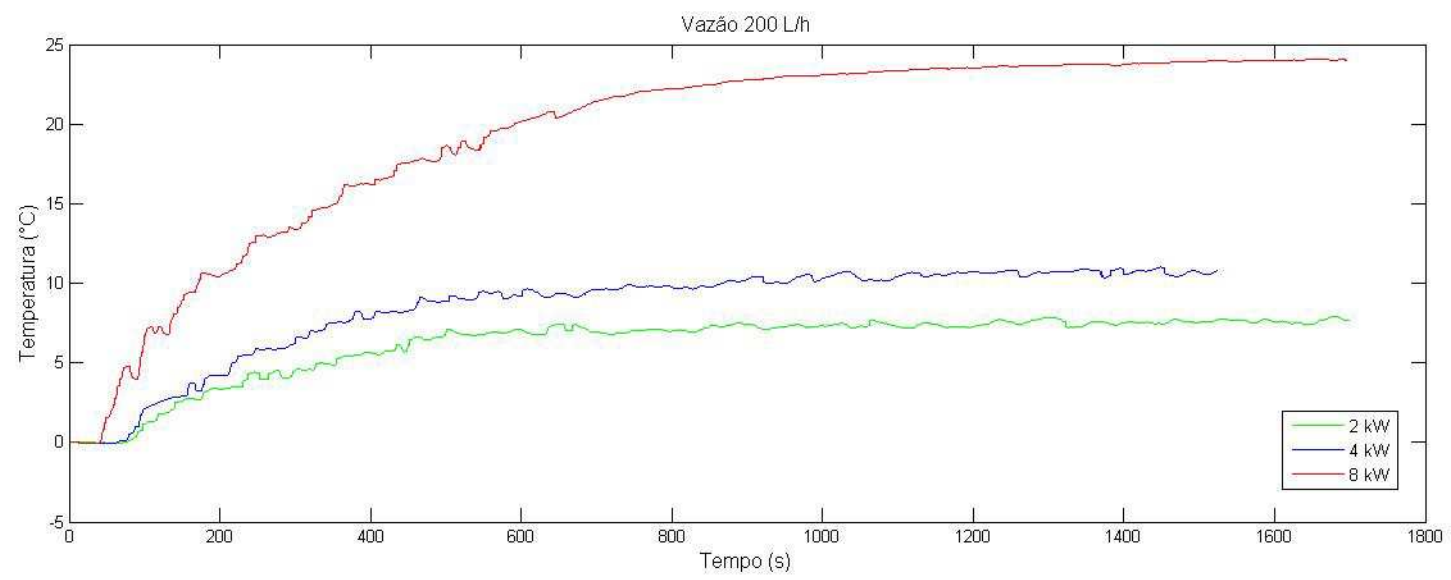

Figura 3 - Temperatura versus tempo na vazão de $200 \mathrm{~L} / \mathrm{h}$ para degraus de 2 , 4 e $8 \mathrm{~kW}$ na potência de entrada.

Como pode ser observado na Figura 3, para o degrau de $2 \mathrm{~kW}$ o valor da temperatura no regime permanente, em variável desvio, foi de $7,74^{\circ} \mathrm{C}$. Logo, o ganho do processo é dado pela Equação 31.

$$
K_{p 1}=\frac{\text { Temperatura no regime permanente }}{\text { degrau de potência }}=\frac{7,74^{\circ} \mathrm{C}}{2 \mathrm{KW}}=3,87 \frac{{ }^{\circ} \mathrm{C}}{\mathrm{kW}}
$$

Para o cálculo da constante de tempo, utilizou-se a relação já descrita anteriormente:

$63,2 \%$ da temperatura no regime permonente $=0,632 \times 7,74^{\circ} \mathrm{C}=4,90^{\circ} \mathrm{C}$

A constante de tempo é o valor do tempo correspondente a temperatura de $4,90^{\circ} \mathrm{C}$, portanto, analisando pelo gráfico da Figura 3, o tempo correspondente é:

$$
4,90^{\circ} \mathrm{C} \leftrightarrow 311 \text { segundos } \rightarrow \tau_{1}=311 \mathrm{~s}
$$

Deve-se atentar para o fato de que os gráficos acima mostram o intervalo de tempo entre o início da medição e a aplicação de cada degrau, já que esse tempo para cada medição foi diferente. Para o cálculo da constante de tempo considerou-se como o tempo inicial (zero) o momento em que cada degrau é aplicado.

Os mesmos cálculos foram feitos para os degraus de 4 e $8 \mathrm{KW}$. A Tabela 1 mostra os valores do ganho e da constante de tempo obtidos para o processo. 
Tabela 1 - Parâmetros das funções de transferência para vazão de 200 L/h.

\begin{tabular}{cccc}
\hline Degrau & $\mathbf{2 ~ k W}$ & $\mathbf{4} \mathbf{~ k W}$ & $\mathbf{8 ~ k W}$ \\
\hline $\mathbf{K p}\left({ }^{\circ} \mathbf{C} / \mathbf{k W}\right)$ & 3,87 & 2,715 & 3,01 \\
$\begin{array}{c}\text { Erro! Fonte } \\
\text { de referência não } \\
\text { encontrada. (s) }\end{array}$ & 311 & 274 & 345 \\
\hline
\end{tabular}

Ainda na vazão de $200 \mathrm{~L} / \mathrm{h}$ foram feitas mais duas replicatas com o degrau de $8 \mathrm{KW}$. A Figura 4 mostra as curvas das triplicatas.

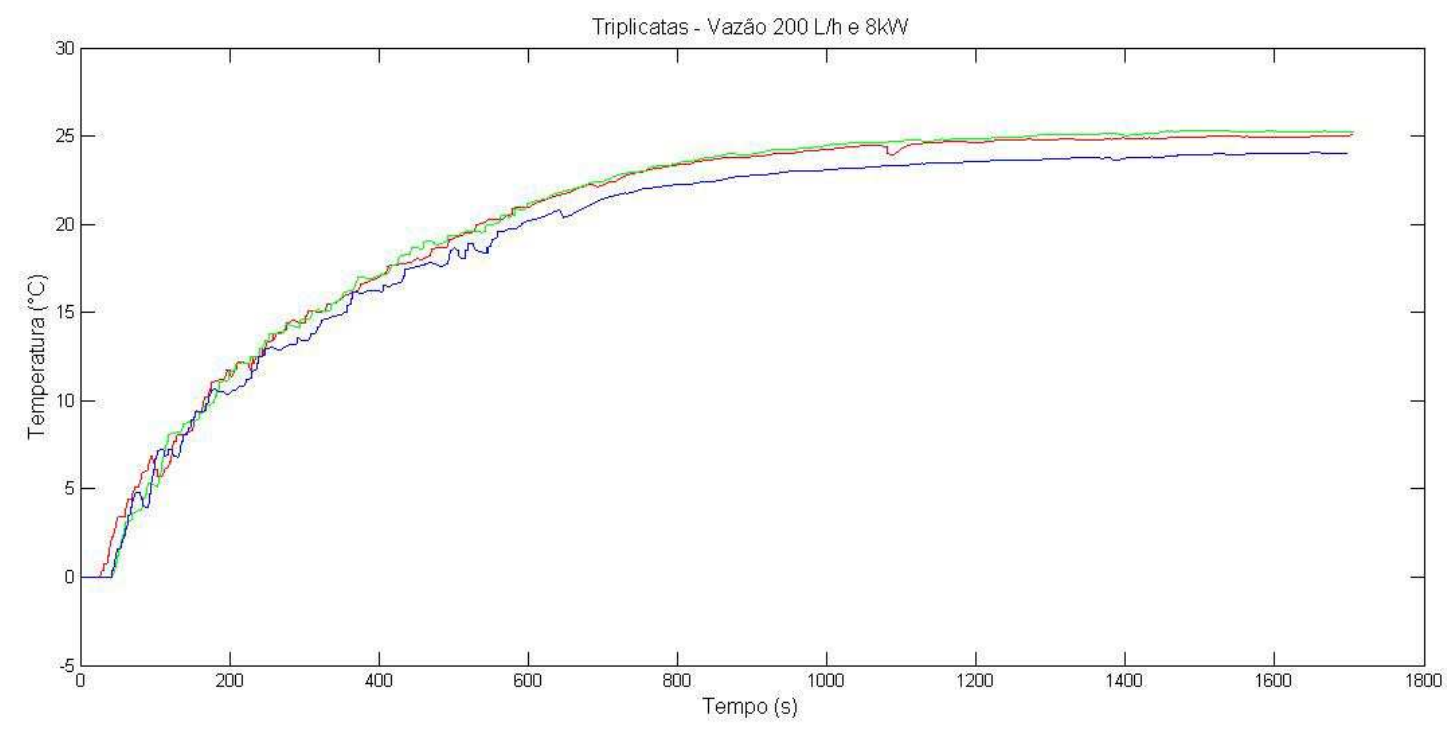

Figura 4- Triplicatas do degrau de 8 KW na vazão de 200L/h.

A Tabela 2 mostra os valores de ganho e da constante de tempo obtidos do processo, todos com vazão de $200 \mathrm{~L} / \mathrm{h}$ e degrau de $8 \mathrm{KW}$, sendo que a curva em azul é referente ao primeiro experimento e as curvas verde e vermelha são referentes às replicatas.

Tabela 2 - Parâmetros das funções de transferência das triplicatas.

\begin{tabular}{llll}
\hline Curva & Azul & Verde & Vermelha \\
\hline
\end{tabular}




\begin{tabular}{rccc}
\hline $\mathbf{K p}\left({ }^{\circ} \mathrm{C} / \mathrm{KW}\right)$ & 3,01 & 3,156 & 3,162 \\
$\begin{array}{r}\text { Erro! Fonte } \\
\text { de referência não } \\
\text { encontrada.(s) }\end{array}$ & 345 & 313 & 324 \\
\hline
\end{tabular}

Devido a ocorrência de muitos ruídos na resposta do sistema, provenientes de distúrbios de natureza desconhecida, foram necessários o tratamento dos dados de resposta da planta ao degrau (média móvel) para a análise do comportamento da temperatura no tanque, utilizando a média móvel no Matlab. Este tratamento atenua as oscilações da curva sem descaracterizar o comportamento do processo, permitindo uma análise mais precisa. A média móvel foi realizada para as vazões ilustradas nas Figuras 5, 6 e 9.

Foram feitos testes na vazão de $400 \mathrm{~L} \cdot \mathrm{h}^{-1}$ de água com degraus de 2 , 4 e $8 \mathrm{~kW}$. As curvas resultantes podem ser melhor observadas pelas linhas da média móvel na Figura 5.

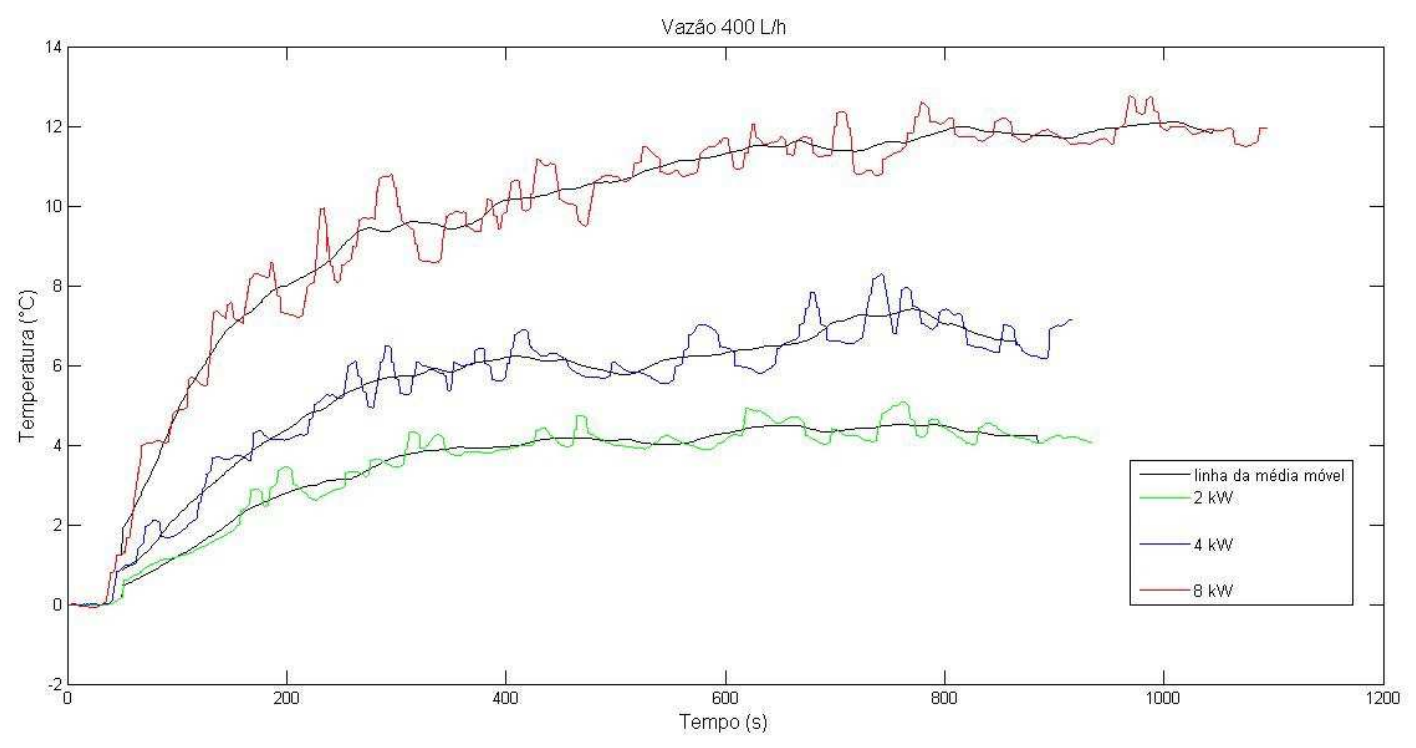

Figura 5 - Temperatura versus tempo na vazão de $400 \mathrm{~L} / \mathrm{h}$.

A Tabela 3 mostra os valores de ganho e da constante de tempo obtidos do processo.

Tabela 3 - Parâmetros das funções de transferência para vazão de $400 \mathrm{~L} / \mathrm{h}$.

\begin{tabular}{llll}
\hline Degrau & $2 \mathrm{KW}$ & $4 \mathrm{KW}$ & $8 \mathrm{KW}$ \\
\hline $\mathrm{Kp}\left({ }^{\circ} \mathrm{C} / \mathrm{KW}\right)$ & 2,28 & 1,8 & 1,5 \\
\hline
\end{tabular}




\begin{tabular}{llll}
\hline $\begin{array}{l}\text { Erro! Fonte de } \\
\text { referência não } \\
\text { encontrada.(s) }\end{array}$ & 237 & 209 & 206 \\
\hline
\end{tabular}

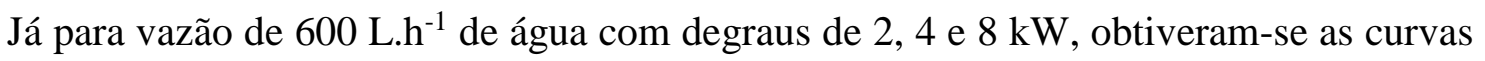
mostradas na Figura 6 e os valores de ganho e constante de tempo mostrados na Tabela 4.

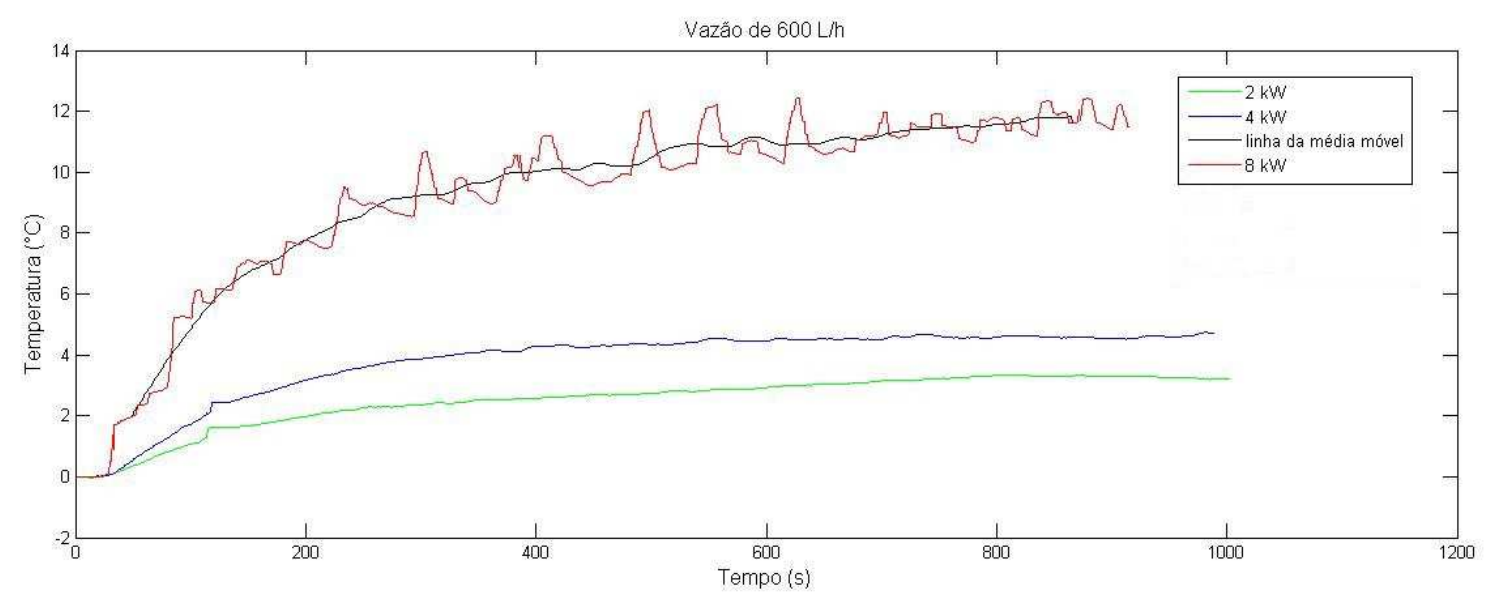

Figura 6 - Temperatura versus tempo na vazão de 600 L/h.

Tabela 4 - Parâmetros das funções de transferência para vazão de 600 L/h.

\begin{tabular}{cccc}
\hline Degrau & $\mathbf{2} \mathbf{~ K W}$ & $\mathbf{4} \mathbf{~ K W}$ & $\mathbf{8} \mathbf{~ W W}$ \\
\hline $\mathbf{K p}\left({ }^{\circ} \mathrm{C} / \mathbf{k W}\right)$ & 1,65 & 1,173 & 1,47 \\
$\begin{array}{c}\text { Erro! Fonte de } \\
\text { referência não } \\
\text { encontrada.(s) }\end{array}$ & 210 & 171 & 172 \\
\hline
\end{tabular}

E, por fim, para vazão máxima de $1000 \mathrm{~L} \cdot \mathrm{h}^{-1}$ com degrau de $8 \mathrm{~kW}$, a curva obtida foi a mostrada na Figura 7. 


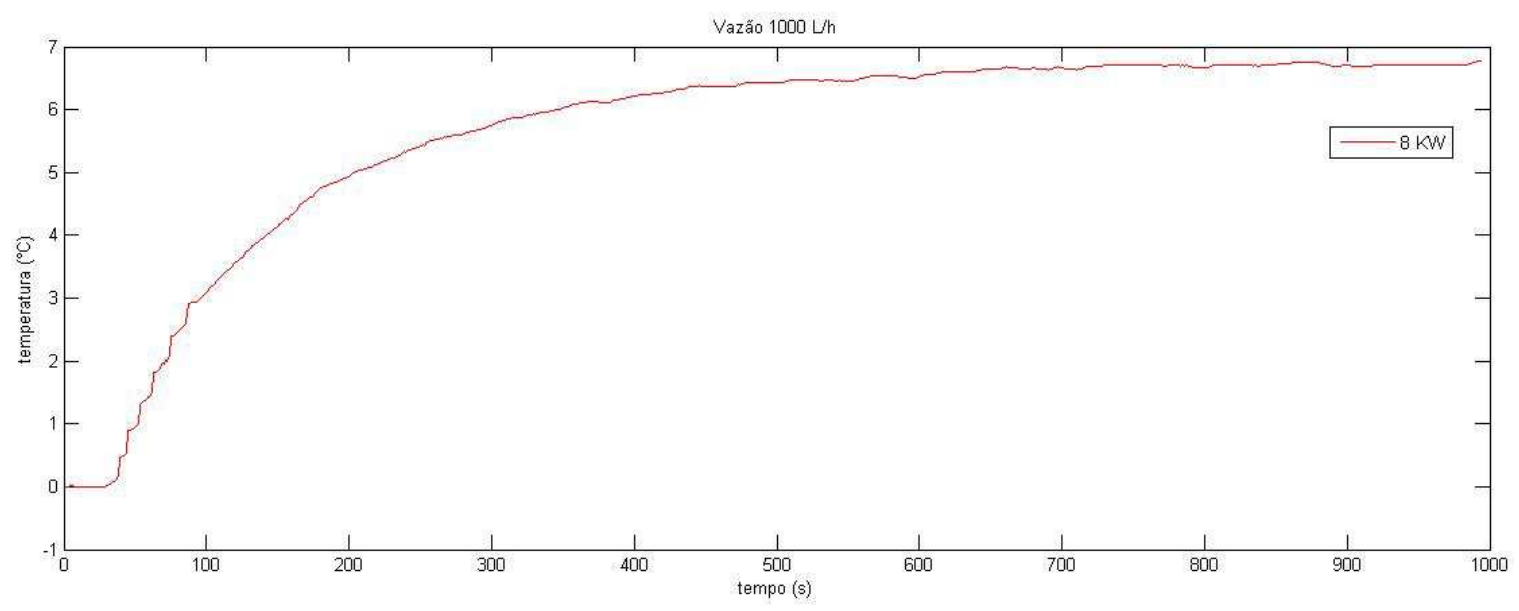

Figura 7 - Temperatura versus tempo na vazão de $1000 \mathrm{~L} / \mathrm{h}$.

Para esta vazão foi realizado somente o degrau máximo de $8 \mathrm{KW}$. O ganho foi de 0,84 ${ }^{\circ} \mathrm{C} / \mathrm{KW}$ e a constante de tempo de 142 segundos.

$\mathrm{Na}$ Tabela 5 estão expostas todas as funções de transferência obtidas dos 12 experimentos.

Tabela 5 - Funções de transferência obtidas com os testes.

\begin{tabular}{|c|c|c|c|}
\hline Vazão\Degrau & $2 \mathrm{~kW}$ & $4 \mathrm{~kW}$ & $8 \mathrm{~kW}$ \\
\hline \multirow[t]{3}{*}{200 L.h-1 } & $\begin{array}{l}\text { Erro! Fonte de } \\
\text { referência não } \\
\text { encontrada. }\end{array}$ & $\begin{array}{l}\text { Erro! Fonte de } \\
\text { referência não } \\
\text { encontrada. }\end{array}$ & $\begin{array}{l}\text { Erro! Fonte de } \\
\text { referência não } \\
\text { encontrada. }\end{array}$ \\
\hline & & & $\begin{array}{c}\text { Erro! Fonte de } \\
\text { referência não } \\
\text { encontrada. }\end{array}$ \\
\hline & & & $\begin{array}{l}\text { Erro! Fonte de } \\
\text { referência não } \\
\text { encontrada. }\end{array}$ \\
\hline 400 L.h-1 & $\begin{array}{l}\text { Erro! Fonte de } \\
\text { referência não } \\
\text { encontrada. }\end{array}$ & $\begin{array}{l}\text { Erro! Fonte de } \\
\text { referência não } \\
\text { encontrada. }\end{array}$ & $\begin{array}{c}\text { Erro! Fonte de } \\
\text { referência não } \\
\text { encontrada. }\end{array}$ \\
\hline 600 L.h-1 & $\begin{array}{l}\text { Erro! Fonte de } \\
\text { referência não } \\
\text { encontrada. }\end{array}$ & $\begin{array}{l}\text { Erro! Fonte de } \\
\text { referência não } \\
\text { encontrada. }\end{array}$ & $\begin{array}{l}\text { Erro! Fonte de } \\
\text { referência não } \\
\text { encontrada. }\end{array}$ \\
\hline
\end{tabular}


Para uma mesma vazão, as funções de transferência encontradas deveriam ser iguais, mas essas diferenças são inevitáveis devido ao método ser empírico. Além disso, certos parâmetros mudam para cada teste feito, como a transferência de calor da água do tanque para o ar externo, que aumenta com o aumento da temperatura do tanque. A soma de todas essas diferenças, associadas a erros que ocorrem naturalmente, levam a essa divergência das funções de transferência encontradas.

Já para vazões diferentes, as funções de transferência mudam, já que maiores vazões requerem uma quantidade de calor maior para aquecer a água no tanque, pois a perda de calor por convecção deve aumentar consideravelmente.

A Tabela 6 mostra as funções de transferência médias para cada valor de vazão de água. Para encontrar as funções de transferência citadas, foi feita uma média aritmética do ganho e da constante de tempo entre as funções de transferência encontradas para cada degrau de potência na mesma vazão.

Tabela 6 - Funções de transferência médias.

\begin{tabular}{ccccc}
\hline Vazão (L/h) & 200 & 400 & 600 & 1000 \\
\hline F.T. média & $\begin{array}{c}\text { Erro! Fonte de } \\
\text { referência não } \\
\text { encontrada. }\end{array}$ & $\begin{array}{c}\text { Erro! Fonte de } \\
\text { referência não } \\
\text { encontrada. }\end{array}$ & $\begin{array}{c}\text { Erro! Fonte de } \\
\text { referência não } \\
\text { encontrada. }\end{array}$ & $\begin{array}{c}\text { Erro! Fonte de } \\
\text { referência não } \\
\text { encontrada. }\end{array}$ \\
\hline
\end{tabular}

\subsection{Validação das Funções de Transferência do Processo}

Nas Figuras 8, 9 e 10, pode-se observar a comparação do experimento com degrau $6 \mathrm{Kw}$, em vermelho, com a média obtida, em verde, e com a função de transferência teórica, em azul, para as vazões de 200, 400 e 600 L.h ${ }^{-1}$, respectivamente. 


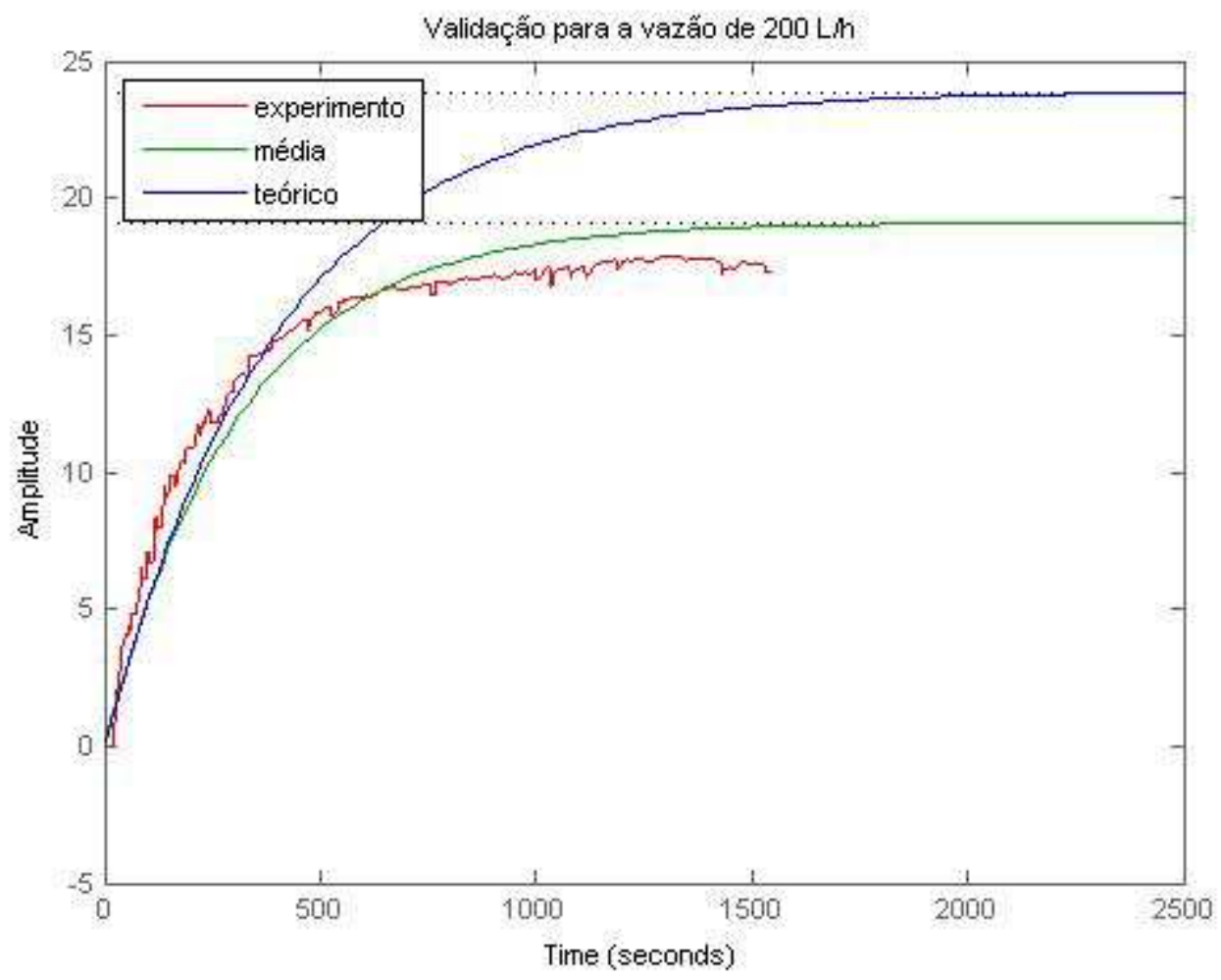

Figura 8 - Validação para vazão de 200 L/h.

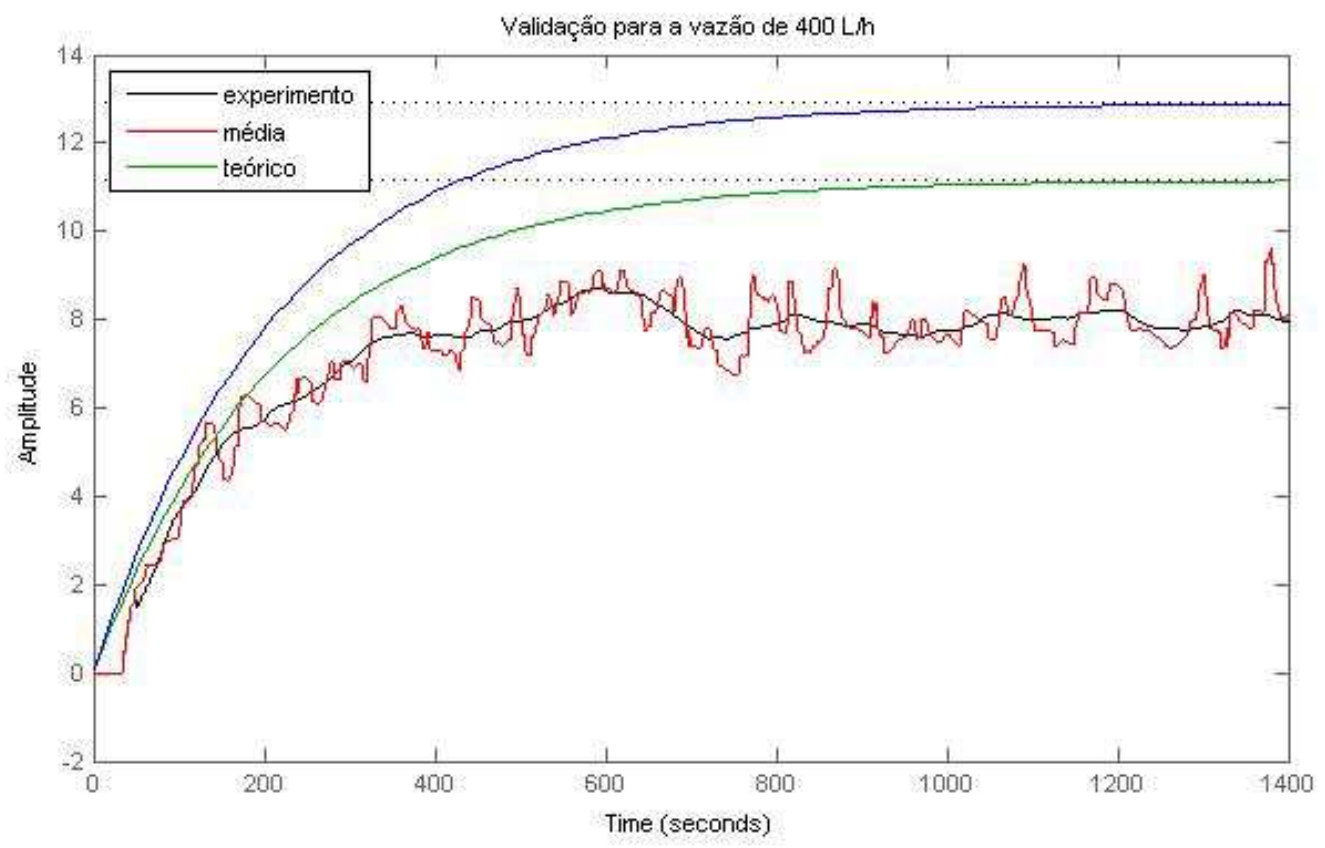

Figura 9 - Validação para vazão de 400 L/h. 


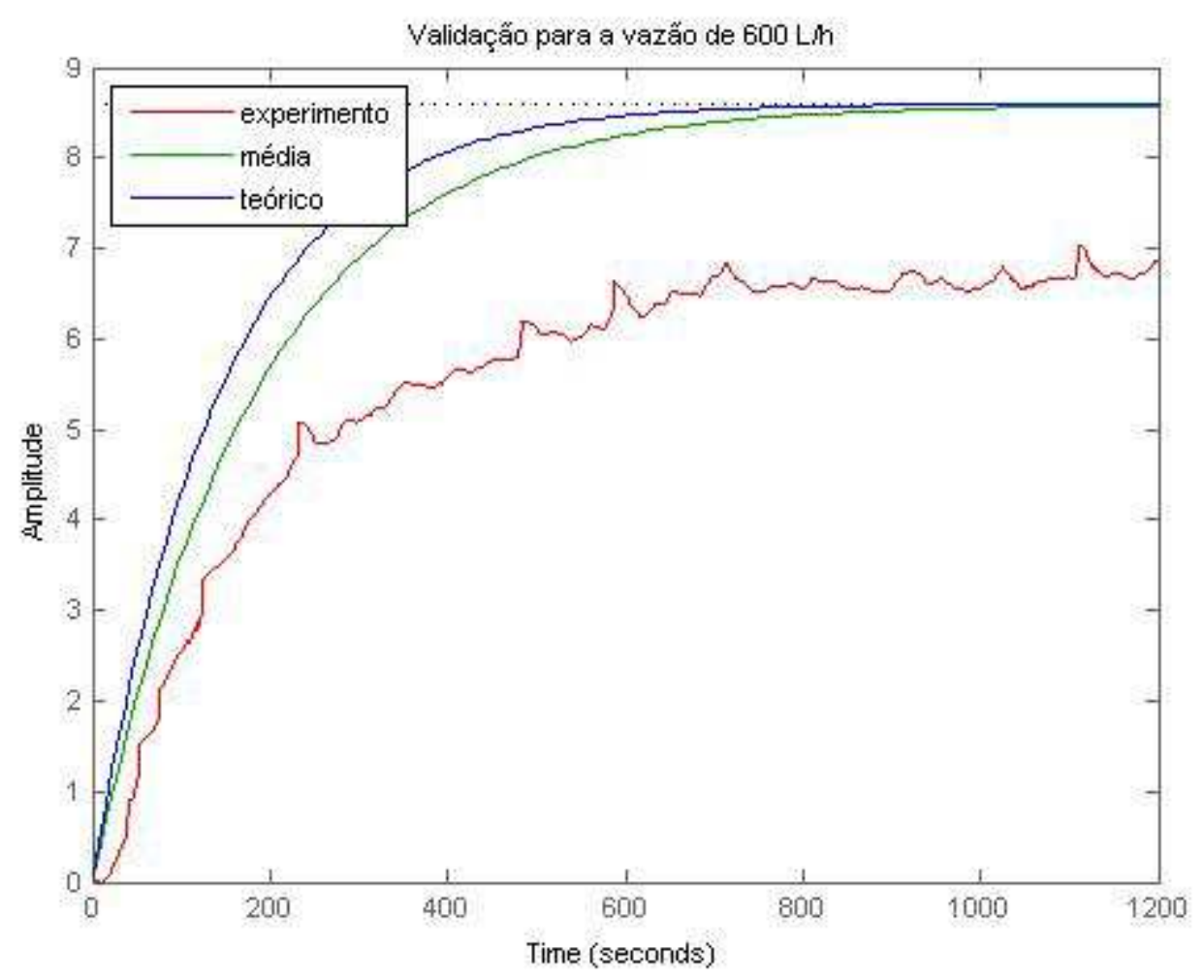

Figura 10 - Validação para vazão de 600 L/h.

Observa-se que os tempos de resposta são muito próximos, variando em torno de 10 a $15 \%$ para as três comparações. Na Figura 8 ocorrem variações nos ganhos, com relação à média, de 26,3\% maior no teórico e 10,53\% menor no experimento. Já na Figura 9 o ganho teórico é $18,2 \%$ maior do que o ganho médio e o experimento $18,2 \%$ menor. No caso da Figura 10 os ganhos do modelo teórico e da média são iguais e o experimento é 18,6\% menor que os anteriores.

A diferença dos ganhos pode ser explicada devido à grande influência de fatores externos como o gradiente de temperatura entre a água no tanque e o ar, que pode influenciar na maior ou menor perda de calor. Desta forma, o modelo teórico tem maior ganho, pois não leva em consideração tal perda de calor, já os experimentos sofrem essa influência, sendo que em dias mais quentes a perda de calor é menor do que em dias mais frios. Além disso, a medida que aumenta o degrau aplicado, ou seja, a potência aplicada, aumenta-se a diferença de temperatura entre a água no tanque e o meio externo e, com isso, aumenta-se a perda de calor.

A fim de demonstrar essa diferença dos ganhos nas funções de transferência, foi calculada a perda de calor no tanque, demostrada na Figura 11. As resistências térmicas representam a dificuldade da transferência de calor por convecção e condução, sendo que as perdas por radiação foram desconsideradas por serem muito baixas. 


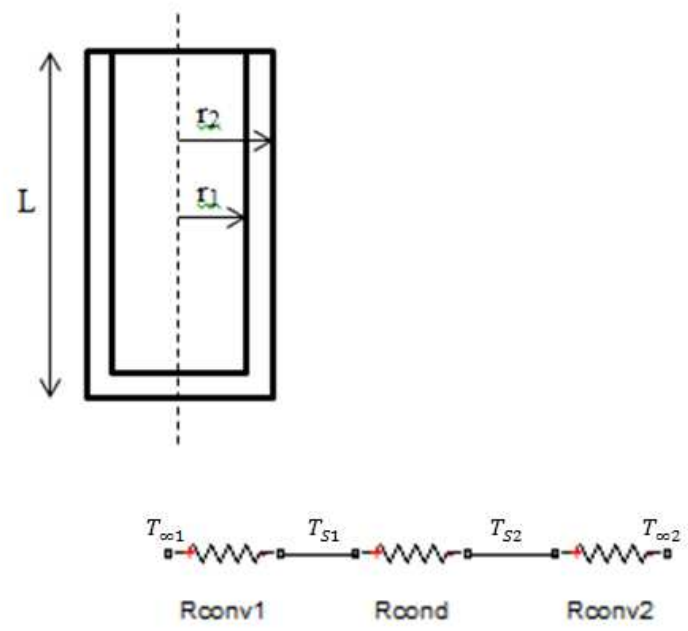

Figura 11 - Representação do tanque de aquecimento e das resistências térmicas.

As resistências são calculadas da seguinte forma:

$$
\begin{aligned}
& R_{\text {conv1 }}=\frac{1}{h_{1} 2 \pi r_{1} L} \\
& R_{\text {cond }}=\frac{\ln \frac{r_{2}}{r_{1}}}{2 \pi k L} \\
& R_{\text {conv2 } 2}=\frac{1}{h_{2} 2 \pi r_{2} L}
\end{aligned}
$$

Onde:

Erro! Fonte de referência não encontrada. é o coeficiente de convecção do fluido interno (água)

Erro! Fonte de referência não encontrada. é o coeficiente de convecção do fluido externo (ar)

Erro! Fonte de referência não encontrada. é o raio interno

Erro! Fonte de referência não encontrada. é o raio externo

Erro! Fonte de referência não encontrada. é a condutividade térmica do material do tanque (aço inox 304)

Erro! Fonte de referência não encontrada. é a altura do tanque

Assim, a perda de calor total será:

$$
q^{\prime \prime}=\frac{T_{\infty 1}-T_{\infty 02}}{R_{T}}
$$


Onde:

Erro! Fonte de referência não encontrada. é a temperatura do fluido interno no regime permanente

Erro! Fonte de referência não encontrada. é a temperatura do fluido externo (Temperatura ambiente)

Erro! Fonte de referência não encontrada. é a resistência total, que como são paralelas, é a soma das resistências individuais.

Este cálculo foi feito para a metade do tanque, como ele é simétrico, multiplica-se por dois e obtém-se a perda total. Pode-se observar pela Tabela 7 que a perda de calor aumenta quando se aplica um degrau maior, numa mesma vazão, e por isso os ganhos observados são diferentes.

Tabela 7 - Cálculo da perda de calor para vazão de $200 \mathrm{~L} / \mathrm{h}$.

\begin{tabular}{llll}
\hline Vazão & $\mathbf{2} \mathbf{k W}$ & $\mathbf{4} \mathbf{k W}$ & $\mathbf{8} \mathbf{k W}$ \\
\hline $\mathbf{2 0 0} \mathbf{L . h}^{-\mathbf{1}}$ & $5,34 \mathrm{~W}$ & $7,36 \mathrm{~W}$ & $16,32 \mathrm{~W}$ \\
\hline
\end{tabular}

\section{CONSIDERAÇÕES FINAIS}

Em função dos testes realizados e dos resultados obtidos, pode-se concluir que a modelagem experimental realizada apresentou resultados satisfatórios e próximos da modelagem teórica, sendo que os valores teóricos são maiores que os experimentais por não considerar a perda de calor mencionada no último tópico, que comprova que quanto maior o degrau, maior a perda de calor e por isso menor o ganho (cerca de até $26 \%$ ). Já a constante do tempo variou de 10 a $15 \%$ para as vazões estudadas. Também foi possível concluir que à medida que se aumenta a vazão, o ganho diminui. Isso era esperado visto que fornecendo uma mesma potência com maior quantidade de água a temperatura alcançada seria menor realmente. Com isso pode-se comprovar a eficiência da metodologia aplicada.

\section{REFERÊNCIAS}

INCROPERA, F. P.; DEWITT, D. P. Fundamentos da Transferência de Calor e de Massa. 6. ed. Rio de Janeiro: LTC, 2008.

KWONG, W. H. Introdução ao Controle de Processos Químicos com MATLAB. Volume 1. São Carlos: EdUFScar, 2012. 212p.

LUYBEN, W. L. Process Modeling, Simulation, and Control for Chemical Engineers. 2. ed. United Stated: McGraw-Hill, 1996. 
OGATA, K. Modern Control Engineering. 5. ed. New Jersey: Prentice Hall, 2010. 894p.

SEBORG, D. A.; EDGAR, T. F.; MELliCHAMP, D. A. Process Dynamics and Control. 2. ed. United States: John Wiley \& Sons, 2003.

SMAR - PD3 - F. Manual de Instruções: Operação e Manutenção - Plantas Didáticas. 2012. Disponívem em: < http://www.smar.com/brasil/produto/pd3-planta-didatica-hartfoundation-fieldbus-e-profibus $>$. Acesso em 15 ago. 2016.

SMAR. PD3 - Planta Didática HART, FOUNDATIONTM Fieldbus e PROFIBUS. Disponível em: <http://www.smar.com/brasil/produto/pd3-planta-didatica-hartfoundation-fieldbus-e-profibus $>$. Acesso em 15 ago. 2016.

STEPHANOPOUloS, G. Chemical Process Control: An Introduction to Theory and Practice. New Jersey: Prentice Hall, 1984. 696p.

SKOCZOWSKI, S. Evaluation Of Order And The Spread Of Time Constants For Aperiodic Processes Using Step Response. Control Engineering Practice, Agosto 1997. Disponível em: < http://www.sciencedirect.com/science/article/pii/S0967066197000993?via\%3Dihub>. Acesso em 11 abril 2017.

AHMED, S.; HUANG, B.; SHAH, S. L. Identification From Step Responses With Transient Initial Conditions. Journal of Process Control, Fevereiro 2008. Disponível em: < http://www.sciencedirect.com/science/article/pii/S0959152407001187>. Acesso em 11 abril 2017.

\title{
DIDACTIC PLANT SMAR PD3: MODELING AND SIMULATION OF HEATING TANK - PART A
}

\author{
C. R. R. de MORAIS ${ }^{1}$, N. B. DOMINGOS ${ }^{2}$, J. V. NICACIO ${ }^{3}$ e A. G. TÔRRES ${ }^{4}$ \\ ${ }^{1}$ Universidade Federal de Viçosa, Departamento de Química \\ ${ }^{2}$ Universidade Federal de Viçosa, Departamento de Química \\ ${ }^{3}$ Universidade Federal de Viçosa, Departamento de Engenharia Agrícola e Ambiental
}


${ }^{4}$ Universidade Federal de Viçosa, Departamento de Engenharia Elétrica E-mail: camilamorais.cr@gmail.com

\begin{abstract}
The PID control (proportional, integral and derivative) is important to ensure the standardization and safety of various industrial chemical processes, as well as its products quality. This paper consists in both theoretical and experimental modeling of the heating tank of the Smar didactic plant III (PD3) for further tuning of the controller to be performed in part $B$ of this study. The didactic plant is located in the Laboratorio de Pesquisa de Processos Industriais e Padrões de Movimentos e Acústica do Departamento de Engenharia Eletrica of the Universidade Federal de Viçosa. We obtained the transfer function parameters from heating process of the tank by using power steps for constant flows of 200, 400, 600 and 1000 L.h $h^{-1}$ and analyzing the data provided by the Process View program. The experimental results were consistent with the theoretical ones, the gains difference up to $26,3 \%$ and the time constants of 10 to $15 \%$, corroborate the efficiency of the applied methodology.
\end{abstract}

KEYWORDS: Modeling; Chemical Processes; Transfer Function; PID controller. 